\title{
IMRT Beam Angle Optimization Using Electromagnetism-Like Algorithm
}

\author{
Humberto Rocha ${ }^{1}$, Ana Maria A.C. Rocha ${ }^{2}$, Joana M. Dias ${ }^{1,3}$, \\ Brigida C. Ferreira ${ }^{4,5}$, and Maria do Carmo Lopes ${ }^{4,5}$ \\ 1 INESC-Coimbra, Rua Antero de Quental, 199 \\ 3000-033 Coimbra, Portugal \\ 2 Departmento de Produção e Sistemas, Algoritmi Research Centre, Universidade do \\ Minho, \\ 4710-057 Braga, Portugal \\ 3 Faculdade de Economia, Universidade de Coimbra, \\ 3004-512 Coimbra, Portugal \\ 4 I3N, Departamento de Física, Universidade de Aveiro, \\ 3810-193 Aveiro, Portugal \\ 5 Serviço de Física Médica, IPOC-FG, EPE, \\ 3000-075 Coimbra, Portugal \\ hrocha@mat.uc.pt, arocha@dps.uminho.pt, joana@fe.uc.pt, \\ brigida@ua.pt, mclopes@ipocoimbra.min-saude.pt
}

\begin{abstract}
The selection of appropriate beam irradiation directions in radiotherapy - beam angle optimization (BAO) problem - is very important for the quality of the treatment, both for improving tumor irradiation and for better organs sparing. However, the BAO problem is still not solved satisfactorily and, most of the time, beam directions continue to be manually selected in clinical practice which requires many trial and error iterations between selecting beam angles and computing fluence patterns until a suitable treatment is achieved. The objective of this paper is to introduce a new approach for the resolution of the BAO problem, using an hybrid electromagnetism-like algorithm with descent search to tackle this highly non-convex optimization problem. Electromagnetismlike algorithms are derivative-free optimization methods with the ability to avoid local entrapment. Moreover, the hybrid electromagnetism-like algorithm with descent search has a high ability of producing descent directions. A set of retrospective treated cases of head-and-neck tumors at the Portuguese Institute of Oncology of Coimbra is used to discuss the benefits of the proposed algorithm for the optimization of the BAO problem.
\end{abstract}

Keywords: Electromagnetism-like mechanism, Descent Search, IMRT, Beam Angle Optimization 


\section{Introduction}

Cancer is one of the most significant health problems worldwide with respect to its incidence and mortality alike. Radiation therapy is, with surgery and chemotherapy, one of the three main treatment approaches for cancer. More than $50 \%$ of all patients diagnosed with cancer, which corresponds to more than 7.6 million patients worldwide, benefit from radiation therapy, either to cure the disease or to palliate symptoms. With this therapy, several beams of ionizing radiation pass through the patient, sent at different incidence directions and centered at the tumor, attempting to sterilize all cancer cells while minimizing the collateral effects on the surrounding healthy organs and tissues. A modern type of radiation therapy is intensity modulated radiation therapy (IMRT), where the radiation beam is modulated by a multileaf collimator. Multileaf collimators enable the transformation of the beam into a grid of smaller beamlets of independent intensities allowing the irradiation of the patient using non-uniform radiation fields from selected angles. A common way to solve the IMRT optimization problems is to use a beamlet-based approach leading to a large-scale mathematical programming problem. Due to the complexity of the whole optimization problem, computation of mathematical algorithms is required to obtain improved solutions.

Typically, radiation is generated by a linear accelerator mounted on a gantry that can rotate along a central axis and is delivered with the patient immobilized on a movable couch. Irradiation from almost any angle, around the tumor, is assured by the combination of the movement of the couch with the rotation of the gantry. However, despite the fact that almost every angle is possible for radiation delivery, the use of coplanar angles is predominant. This is a way to simplify an already complex problem, and the angles considered lay in the plane of the rotation of the gantry around the patient. Furthermore, regardless of the evidence presented in the literature that selecting appropriate radiation beam incidence directions - beam angle optimization (BAO) problem - can lead to a plan's quality improvement $[7,14]$, in clinical practice, most of the time, beam directions continue to be either manually selected by the treatment planner in a time-consuming trial and error iterative process or patients are irradiated using evenly spaced coplanar beams. The difficulty of solving the BAO problem, a highly non-convex problem with many local minima on a large search space [6], is one possible justification for the current clinical practice.

In this paper we present the benefits of using a hybrid electromagnetismlike algorithm with descent search for the optimization of the highly non-convex BAO problem. A set of clinical cases of head-and-neck tumors treated at the Portuguese Institute of Oncology of Coimbra is used to discuss the potential of this approach in the optimization of the BAO problem. The paper is organized as follows. In the next section we describe the BAO problem formulation. Section 3 briefly presents the hybrid electromagnetism-like algorithm with descent search. Computational tests using clinical examples of head-and-neck cases are presented in Section 4. In the last section we have the conclusions. 


\section{Beam Angle Optimization in IMRT Treatment Planning}

The BAO problem is a quite difficult problem to solve since it is a highly nonconvex optimization problem with many local minima - see Fig. 1. Except for rare exceptions, where the BAO problem is addressed as a non-convex nonlinear problem, for the vast majority of previous work on beam angle optimization, the continuous $\left[0^{\circ}, 360^{\circ}\right]$ gantry angles are discretized into equally spaced beam directions with a given angle increment, such as 5 or 10 degrees, where exhaustive searches are performed directly or guided by a variety of different heuristics including simulated annealing [5], genetic algorithms [9], particle swarm optimization [13] or other heuristics incorporating a priori knowledge of the problem [16]. Although those global heuristics can theoretically avoid local optima, globally optimal or even clinically better solutions can not be obtained without a large number of objective function evaluations. On the other hand, the use of single-beam metrics has been a popular approach to address the BAO problem as well, e.g., the concept of beam's-eye-view [15]. The concept is similar to a bird's eye view, where the object being viewed is the tumor as seen from a beam. The bigger the area of the tumor and the smaller the area of the surrounding organs is seen by the beam, the better candidate the beam is to be used in the treatment plan. Despite the computational time efficiency of these approaches, the quality of the solutions proposed cannot be guaranteed since the interplay between the selected beam directions is ignored. Many other attempts to address the BAO problem can be found in literature. Ehrgott et al. [10] discuss a mathematical framework that unifies the approaches found in literature. Aleman et al. [2] propose a response surface approach and include non-coplanar angles in beam orientation optimization. Lee et al. [12] suggest a mixed integer programming (MIP) approach for simultaneously determining an optimal intensity map and optimal beam angles for IMRT delivery. Schreibmann et al. [23] propose a hybrid multiobjective evolutionary optimization algorithm for IMRT inverse planning and apply it to the optimization of the number of incident beams, their orientations and intensity profiles. Other approaches include maximal geometric separation of treatment beams [7] or gradient searches [6].

The BAO problem is the first problem that arises in treatment planning, but its optimal solution is highly dependent on the optimal solution of the fluence map optimization (FMO) problem - the problem of deciding what are the optimal radiation intensities associated with each set of beam angles. Many of the previous BAO studies are based on a variety of scoring methods or approximations of the FMO to gauge the quality of the beam angle set leading to beam angle sets with no guarantee of optimality and questionable reliability since it has been extensively reported that optimal beam angles for IMRT are often nonintuitive [24]. The optimal solution of the FMO problem has been used to drive the BAO problem $[1,6,23]$ including in our works $[9,19,20,21]$. Our approach for modeling the $\mathrm{BAO}$ problem uses the optimal solution value of the FMO problem as the measure of the quality for a given beam angle set. Thus, we will present the formulation of the $\mathrm{BAO}$ problem followed by the formulation of the FMO 

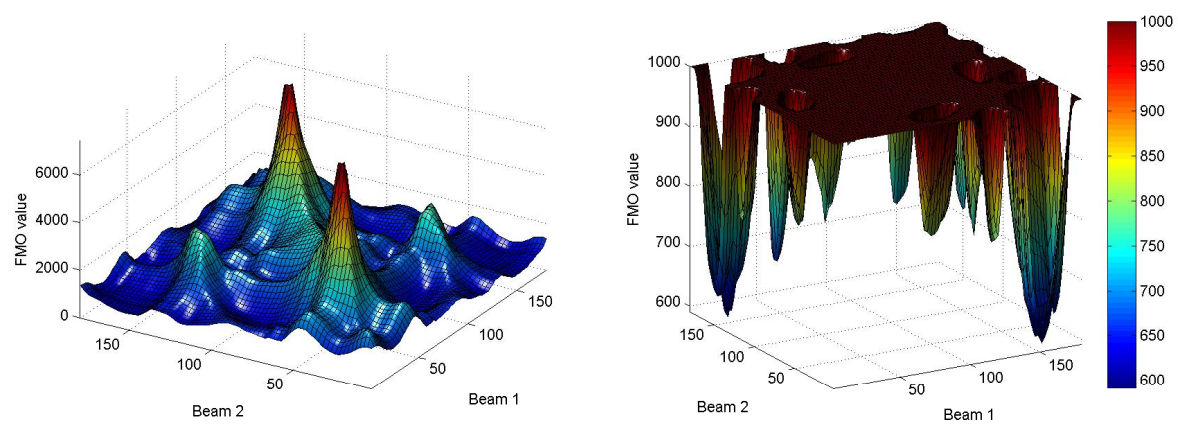

Fig. 1. 2-beam BAO surface (left) and truncated surface (right) to highlight the many local minima.

problem we used. Here, we will assume that the number of beam angles is defined a priori by the treatment planner and that all the radiation directions lie on the same plane.

\subsection{BAO Model}

Let us consider $n$ to be the fixed number of (coplanar) beam directions, i.e., $n$ beam angles are chosen on a circle around the CT-slice of the body that contains the isocenter (usually the center of mass of the tumor). In our formulation, instead of a discretized sample, all continuous $\left[0^{\circ}, 360^{\circ}\right]$ gantry angles will be considered. Since the angle $-5^{\circ}$ is equivalent to the angle $355^{\circ}$ and the angle $365^{\circ}$ is the same as the angle $5^{\circ}$, we can avoid a bounded formulation. A simple formulation for the BAO problem is obtained by selecting an objective function such that the best set of beam angles is obtained for the function's minimum:

$$
\begin{aligned}
& \min f\left(\theta_{1}, \ldots, \theta_{n}\right) \\
& \text { s.t. }\left(\theta_{1}, \ldots, \theta_{n}\right) \in \mathbb{R}^{n} .
\end{aligned}
$$

Here, for the reasons stated before, the objective $f\left(\theta_{1}, \ldots, \theta_{n}\right)$ that measures the quality of the set of beam directions $\theta_{1}, \ldots, \theta_{n}$ is the optimal value of the FMO problem for each fixed set of beam directions. The FMO model used is presented next.

\section{$2.2 \quad$ FMO Model}

In order to solve the FMO problem, i.e., to determine optimal fluence maps, the radiation dose distribution deposited in the patient needs to be assessed accurately. Each structure's volume is discretized into small volume elements (voxels) and the dose is computed for each voxel considering the contribution of each beamlet. Typically, a dose matrix D is constructed from the collection of 
all beamlet weights, by indexing the rows of $\mathrm{D}$ to each voxel and the columns to each beamlet, i.e., the number of rows of matrix $\mathrm{D}$ equals the number of voxels $\left(N_{v}\right)$ and the number of columns equals the number of beamlets $\left(N_{b}\right)$ from all beam directions considered. Therefore, using matrix format, we can say that the total dose received by the voxel $i$ is given by $\sum_{j=1}^{N_{b}} D_{i j} w_{j}$, with $w_{j}$ the weight of beamlet $j$. Usually, the total number of voxels is large, reaching the tens of thousands, which originates large-scale problems. This is one of the main reasons for the difficulty of solving the FMO problem.

For a given beam angle set, an optimal IMRT plan is obtained by solving the FMO problem - the problem of determining the optimal beamlet weights for the fixed beam angles. Many mathematical optimization models and algorithms have been proposed for the FMO problem, including linear models [22], mixed integer linear models [11] and nonlinear models [1]. Here, we will use this latter approach that penalizes each voxel according to the square difference of the amount of dose received by the voxel and the amount of dose desired/allowed for the voxel. This formulation yields a quadratic programming problem with only linear non-negativity constraints on the fluence values [22]:

$$
\begin{aligned}
& \min _{w} \sum_{i=1}^{N_{v}} \frac{1}{v_{S}}\left[\underline{\lambda}_{i}\left(T_{i}-\sum_{j=1}^{N_{b}} D_{i j} w_{j}\right)_{+}^{2}+\bar{\lambda}_{i}\left(\sum_{j=1}^{N_{b}} D_{i j} w_{j}-T_{i}\right)_{+}^{2}\right] \\
& \text { s.t. } \quad w_{j} \geq 0, j=1, \ldots, N_{b},
\end{aligned}
$$

where $T_{i}$ is the desired dose for voxel $i$ of the structure $v_{S}, \underline{\lambda}_{i}$ and $\bar{\lambda}_{i}$ are the penalty weights of underdose and overdose of voxel $i$, and $(\cdot)_{+}=\max \{0, \cdot\}$. This nonlinear formulation implies that a very small amount of underdose or overdose may be accepted in clinical decision making, but larger deviations from the desired/allowed doses are decreasingly tolerated [1].

The FMO model is used as a black-box function and the conclusions drawn regarding BAO coupled with this nonlinear model are valid also if different FMO formulations are considered.

\section{Electromagnetism-Like Algorithm}

The Electromagnetism-like (EM) algorithm, developed by Birbil and Fang [3], is a population-based stochastic search method for global optimization that mimics the behavior of electrically charged particles. The method uses an attractionrepulsion mechanism to move a population of points towards optimality. The EM algorithm is designed for solving optimization problems in the following form:

$$
\begin{array}{cl}
\min & f(x) \\
\text { s.t. } & x \in \Omega,
\end{array}
$$

where $f: \mathbb{R}^{n} \rightarrow \mathbb{R}$ is a nonlinear continuous function and $\Omega=\left\{x \in \mathbb{R}^{n}: l b \leq\right.$ $x \leq u b\}$ is a bounded feasible region. We do not assume the objective function is convex and we consider it may have many local minima in the feasible region. 
The EM algorithm simulates the electromagnetism theory of physics by considering each point in the population as an electrical charge that is released to the space. The charge of each point is related to the objective function value and determines the magnitude of attraction of the point over the others in the population. The better the objective function value, the higher the magnitude of attraction. The charges are used to find a direction for the movement of each points. The regions that have higher attraction will signal other points to move towards them. In addition, a repulsion mechanism is also introduced to explore new regions for even better solutions [3,4].

The EM algorithm is described in Algorithm 1 and comprises four main procedures: "Initialization", "Compute Force", "Move Points" and "Local Search".

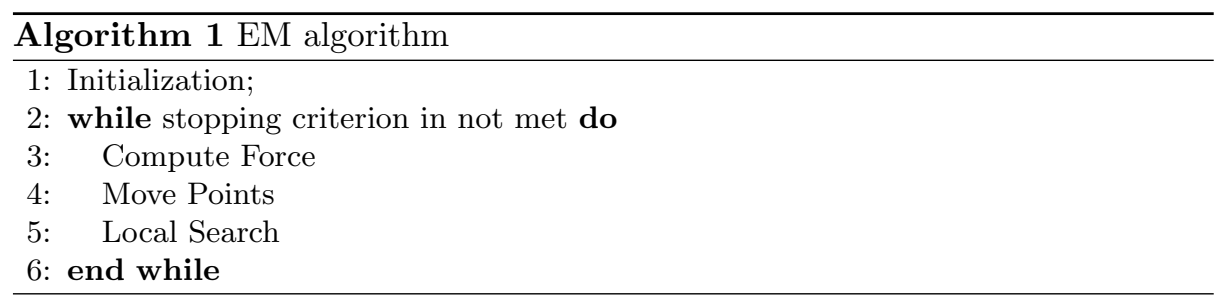

The "Initialization" procedure starts by randomly generating a sample of $m$ points. Each point is uniformly distributed between the lower and upper bounds. Then, the objective function value for each point is calculated and the best point of the population, $x^{\text {best }}$, is identified as well as its corresponding objective function value $f^{\text {best }}$. After the initialization of the population, and identification of the best point of the population, the other procedures are executed until the stopping criterion is met.

In the "Compute Force" procedure, each particle's charge is calculated by the following formula:

$$
q^{i}=\exp \left(-n \frac{f\left(x^{i}\right)-f\left(x^{b e s t}\right)}{\sum_{k=1}^{m}\left(f\left(x^{k}\right)-f\left(x^{\text {best }}\right)\right)}\right), i=1, \ldots, m .
$$

that determines the power of attraction or repulsion for the point $x^{i}$. In this way the points that have better objective function values possess higher charges. After the charge calculation, the total force vector $F^{i}$ on each point $x^{i}$ is then calculated by adding the individual component forces, $F_{j}^{i}$, between any pair of points $x^{i}$ and $x^{j}$,

$$
F^{i}=\sum_{j=1, j \neq i}^{m} F_{j}^{i}=\left\{\begin{array}{l}
\left(x^{j}-x^{i}\right) \frac{q^{i} q^{j}}{\left\|x^{j} x^{i}\right\|^{2}} \text { if } f\left(x^{j}\right)<f\left(x^{i}\right) \text { (attraction) } \\
\left(x^{i}-x^{j}\right) \frac{q^{i} q^{j}}{\left\|x^{j}-x^{i}\right\|^{2}} \text { if } f\left(x^{j}\right) \geq f\left(x^{i}\right) \text { (repulsion) }
\end{array},\right.
$$

for $i=1,2, \ldots, m$. 
The "Move Points" procedure uses the total force vector, $F^{i}$, to move the point $x^{i}$ in the direction of the force by a random step length $\lambda$. The best point, $x^{\text {best }}$, is not moved. To maintain feasibility, the force exerted on each point is normalized and scaled by the allowed range of movement towards the lower bound or the upper bound of the set $\Omega$, for each coordinate. Thus, for $i=1,2, \ldots, m$ and $i \neq$ best

$$
x_{k}^{i}=\left\{\begin{array}{l}
x^{i}+\lambda \frac{F^{i}}{\left\|F^{i}\right\|}\left(u-x^{i}\right) \text { if } F^{i}>0 \\
x^{i}+\lambda \frac{F^{i}}{\left\|F^{i}\right\|}\left(x^{i}-l\right) \text { otherwise }
\end{array} .\right.
$$

The step length $\lambda$ is assumed to be an uniformly distributed random variable in $[0,1]$.

Finally, a local refinement around the best point of the population is done by the "Local search" procedure in order to improve the accuracy of EM $[3,4,17,18]$. This procedure implements a simple random line search algorithm, using the maximum feasible step length $s_{\max }=\delta_{\text {local }}(\max [u-l])$, with $\delta_{\text {local }}>0$, to guarantee that the local search always generates feasible points. A trial point $y$, componentwise defined by $y=x^{\text {best }}+\lambda s_{\max }$, with $\lambda \sim U[0,1]$, is computed. If $y$ improves over $x^{\text {best }}$ within a maximum number of local iterations, $x^{\text {best }}$ is updated. See $[3,17,18]$ for details.

\section{Computational Results for Head-and-Neck Clinical Examples}

The hybrid EM algorithm was tested using two clinical examples of retrospective treated cases of head-and-neck tumors at the Portuguese Institute of Oncology of Coimbra (IPOC). In general, the head-and-neck region is a complex area to treat with radiotherapy due to the large number of sensitive organs in this region (e.g., eyes, mandible, larynx, oral cavity, etc.). For simplicity, in this study, the organs at risk (OARs) used for treatment optimization were limited to the spinal cord, the brainstem and the parotid glands. The spinal cord and the brainstem are some of the most critical OARs in the head-and-neck tumor cases. These are serial organs, i.e., organs such that if only one subunit is damaged, the whole organ functionality is compromised. Therefore, if the tolerance dose is exceeded, it may result in functional damage to the whole organ. Thus, it is extremely important not to exceed the tolerance dose prescribed for these type of organs. Other than the spinal cord and the brainstem, the parotid glands are also important OARs. The parotid gland is the largest of the three salivary glands. A common complication due to parotid glands irradiation is xerostomia (the medical term for dry mouth due to lack of saliva). This decreases the quality of life of patients undergoing radiation therapy of head-and-neck, causing difficulties to swallow. The parotids are parallel organs, i.e., if a small volume of the organ is damaged, the rest of the organ functionality may not be affected. Their tolerance dose depends strongly on the fraction of the volume irradiated. Hence, if only a small fraction of the organ is irradiated the tolerance dose is much higher than 
Table 1. Prescribed doses for all the structures considered for IMRT optimization.

\begin{tabular}{lccc}
\hline Structure & \multicolumn{3}{c}{ Mean dose } \\
\hline Spinal cord & - & $45 \mathrm{~Gy}$ & - \\
Brainstem & - & $54 \mathrm{~Gy}$ & - \\
Left parotid & $26 \mathrm{~Gy}$ & - & - \\
Right parotid & $26 \mathrm{~Gy}$ & - & - \\
PTV1 & - & - & $70.0 \mathrm{~Gy}$ \\
PTV2 & - & - & $59.4 \mathrm{~Gy}$ \\
Body & - & $80 \mathrm{~Gy}$ & - \\
\hline
\end{tabular}

if a larger fraction is irradiated. Thus, for these parallel structures, the organ mean dose is generally used instead of the maximum dose as an objective for inverse planning optimization. The tumor to be treated plus some safety margins is called planning target volume (PTV). For the head-and-neck cases in study it was separated in two parts with different prescribed doses: PTV1 and PTV2. The prescription dose for the target volumes and tolerance doses for the OARs considered in the optimization are presented in Table 1.

Our tests were performed on a 2.66Ghz Intel Core Duo PC with 3 GB RAM. In order to facilitate convenient access, visualization and analysis of patient treatment planning data, as well as dosimetric data input for treatment plan optimization research, the computational tools developed within MATLAB and CERR - a freeware computational environment for radiotherapy research [8] - are used widely for IMRT treatment planning research. The patients' CT sets and delineated structures are exported via Dicom RT to CERR. We used CERR 3.2.2 version and MATLAB 7.4.0 (R2007a). An automatized procedure for dose computation for each given beam angle set was developed, instead of the traditional dose computation available from IMRTP module accessible from CERR's menubar. This automatization of the dose computation was essential for integration in our $\mathrm{BAO}$ algorithm. To address the convex nonlinear formulation of the FMO problem we used a trust-region-reflective algorithm (fmincon) of MATLAB 7.4.0 (R2007a) Optimization Toolbox. In order to obtain a solution in a clinically acceptable computational time (one night), the population size of the EM algorithm was limited to 10 and the maximum number of function evaluations was set to 200. The equispaced solution was always included in the initial population.

Treatment plans with five to nine equispaced coplanar beams are used at IPOC and are commonly used in practice to treat head-and-neck cases [1]. Therefore, treatment plans of seven coplanar orientations were obtained using the hybrid EM algorithm. For each patient, ten runs of the EM algorithm were performed. Each run took 8 to 10 hours of computational time. Table 2 presents the computational results, considering the improvement of the objective function value of the final solution ( $f E M$ ) when compared with the equidistant solution (fequi), the beam angle configuration typically used in clinical practice. 
Table 2. FMO value (mean) improvement obtained by $E M$ compared with the typical equispaced coplanar treatment plans equi.

\begin{tabular}{ccccc}
\hline Case & fequi & Mean fEM & \% decrease & Std \\
\hline 1 & 73.75 & 68.67 & $6.88 \%$ & 0.95 \\
2 & 161.22 & 151.94 & $5.76 \%$ & 0.49 \\
\hline
\end{tabular}

Despite the improvement in FMO value, the quality of the results can be perceived considering a variety of metrics. A metric usually used for plan evaluation is the volume of PTV that receives $95 \%$ of the prescribed dose. Typically, $95 \%$ of the PTV volume is required. The occurrence of coldspots, less than $93 \%$ of PTV volume receives the prescribed dose, and the existence of hotspots, the percentage of the PTV volume that receives more than $110 \%$ of the prescribed dose, are other measures usually used to evaluate target coverage. Mean and/or maximum doses of OARs are usually displayed to verify organ sparing.

Table 3. Target coverage obtained by treatment plans.

\begin{tabular}{|c|c|c|c|c|}
\hline Case & Target coverage & EMbest & EMworst & equi \\
\hline \multirow{6}{*}{1} & PTV1 at $95 \%$ volume & \multicolumn{3}{|c|}{ 67.28 Gy 67.28 Gy 67.43 Gy } \\
\hline & PTV1 $\%>93 \%$ of $\mathrm{Rx}(\%)$ & 99.33 & 99.59 & 99.53 \\
\hline & PTV $1 \%>110 \%$ of $\mathrm{Rx}(\%)$ & 0.00 & 0.00 & 0.00 \\
\hline & PTV2 at $95 \%$ volume & \multicolumn{3}{|c|}{56.98 Gy 56.73 Gy 56.42 Gy } \\
\hline & PTV $2 \%>93 \%$ of $\mathrm{Rx}(\%)$ & 96.75 & 96.49 & 96.11 \\
\hline & PTV $2 \%>110 \%$ of $\operatorname{Rx}(\%)$ & 5.66 & 5.80 & 5.93 \\
\hline \multirow{6}{*}{2} & PTV1 at $95 \%$ volume & \multicolumn{3}{|c|}{ 65.08 Gy 65.03 Gy 65.08 Gy } \\
\hline & PTV1 $\%>93 \%$ of $\mathrm{Rx}(\%)$ & 94.68 & 94.73 & 94.59 \\
\hline & PTV1 $\%>110 \%$ of $\mathrm{Rx}(\%)$ & 0.00 & 0.00 & 0.00 \\
\hline & PTV2 at $95 \%$ volume & \multicolumn{3}{|c|}{ 56.28 Gy 56.38 Gy 56.08 Gy } \\
\hline & PTV $2 \%>93 \%$ of $\mathrm{Rx}(\%)$ & 95.97 & 96.08 & 95.86 \\
\hline & PTV $2 \%>110 \%$ of $\operatorname{Rx}(\%)$ & 19.14 & 18.97 & 19.04 \\
\hline
\end{tabular}

The results regarding targets coverage are presented in Table 3. In clinical practice, in order to obtain a solution in a clinical acceptable time frame, we can only run the EM algorithm once. Although the small standard deviation obtained, results are presented for the best solution achieved and also for the worst, denoted EMbest and EMworst. We can verify that optimized treatment plans consistently obtained slightly better target coverage numbers compared to equidistant treatment plans, denoted equi. Organ sparing results are shown in Table 4. All the treatment plans fulfill the maximum dose requirements for the spinal cord and the brainstem. However, as expected, the main differences reside in parotid sparing. The optimized treatment plans clearly improve the usually 
Table 4. OARs sparing obtained by treatment plans.

\begin{tabular}{|c|c|c|c|c|c|c|c|}
\hline \multirow[b]{2}{*}{ Case } & \multirow[b]{2}{*}{ OAR } & \multicolumn{3}{|c|}{ Mean Dose (Gy) } & \multicolumn{3}{|c|}{ Max Dose (Gy) } \\
\hline & & EMbest & EMworst & equi & EMbest & EMworst & equi \\
\hline \multirow{4}{*}{1} & Spinal cord & - & - & - & 42.53 & 44.42 & 44.27 \\
\hline & Brainstem & - & - & - & 52.98 & 53.93 & 53.18 \\
\hline & Left parotid & 26.64 & 26.74 & 27.21 & - & - & - \\
\hline & Right parotid & 25.41 & 25.59 & 27.03 & - & - & - \\
\hline \multirow{4}{*}{2} & Spinal cord & - & - & - & 41.43 & 41.03 & 42.63 \\
\hline & Brainstem & - & - & - & 47.53 & 48.78 & 48.08 \\
\hline & Left parotid & 25.89 & 26.99 & 27.38 & - & - & - \\
\hline & Right parotid & 28.34 & 28.91 & 29.14 & - & - & - \\
\hline
\end{tabular}

clinically used equispaced treatment plans. Most important is to remark that, despite the best run achieved better results, the worst run managed to obtain, as well, a better parotid sparing than the typical equispaced configuration.

\section{Conclusions}

The BAO problem is a continuous global highly non-convex optimization problem known to be extremely challenging and yet to be solved satisfactorily. This paper proposes an alternative approach to the BAO problem using a hybrid electromagnetism-like algorithm with descent search, which is yet another step on the quest that may take us closer to find a better solution in a clinical acceptable time. The hybrid electromagnetism-like algorithm with descent search has already proved to be a suitable approach for the resolution of non-convex problems due to its faster progress towards optimality together with a higher consistency solution. For the clinical cases retrospectively tested, the use of this approach lead to solutions with better objective function value than the typical equispaced solution using a limited number of function evaluations. The improvement of the solutions in terms of objective function value corresponded, for the head-and-neck cases tested, to high quality treatment plans with better target coverage and with improved organ sparing.

Acknowledgments. This work was supported by QREN under Mais Centro (CENTRO-07-0224-FEDER-002003) and FEDER funds through the COMPETE program and Portuguese funds through FCT under project grant PTDC/EIACCO/121450/2010. This work has also been partially supported by FCT under project grant PEst-OE/EEI/UI308/2014 and in the scope of the project PEstOE/EEI/UI0319/2014. The work of H. Rocha was supported by the European social fund and Portuguese funds from MCTES. 


\section{References}

1. Aleman, D.M., Kumar, A., Ahuja, R.K., Romeijn, H.E., Dempsey, J.F.: Neighborhood search approaches to beam orientation optimization in intensity modulated radiation therapy treatment planning. J. Global Optim. 42, 587-607 (2008)

2. Aleman, D.M., Romeijn, H.E., Dempsey, J.F.: A response surface approach to beam orientation optimization in intensity modulated radiation therapy treatment planning. INFORMS J. Comput.: Computat. Biol. Med. Appl. 21, 62-76 (2009)

3. Birbil, S.I., Fang, S.-C.: An electromagnetism-like mechanism for global optimization. J. Global Optim. 25, 263-282 (2003)

4. Birbil, S.I., Fang, S.C., Sheu, R.L.: On the convergence of a population-based global optimization algorithm. J. Global Optim. 30, 301-318 (2004)

5. Bortfeld, T., Schlegel, W.: Optimization of beam orientations in radiation therapy: some theoretical considerations. Phys. Med. Biol. 38, 291-304 (1993)

6. Craft, D.: Local beam angle optimization with linear programming and gradient search. Phys. Med. Biol. 52, 127-135 (2007)

7. Das, S.K., Marks, L.B.: Selection of coplanar or non coplanar beams using threedimensional optimization based on maximum beam separation and minimized nontarget irradiation. Int. J. Radiat. Oncol. Biol. Phys. 38, 643-655 (1997)

8. Deasy, J.O., Blanco, A.I., Clark, V.H.: CERR: A Computational Environment for Radiotherapy Research. Med. Phys. 30, 979-985 (2003)

9. Dias, J., Rocha, H., Ferreira, B.C., Lopes, M.C.: A genetic algorithm with neural network fitness function evaluation for IMRT beam angle optimization. Cent. Eur. J. Oper. Res. (in press) doi:10.1007/s10100-013-0289-4

10. Ehrgott, M., Holder, A., Reese, J.: Beam selection in radiotherapy design. Linear Algebra Appl. 428, 1272-1312 (2008)

11. Lee, E.K., Fox, T., Crocker, I.: Integer programming applied to intensity-modulated radiation therapy treatment planning. Ann. Oper. Res. 119, 165-181 (2003)

12. Lee, E.K., Fox, T., Crocker, I.: Simultaneous beam geometry and intensity map optimization in intensity-modulated radiation therapy. Int. J. Radiat. Oncol. Biol. Phys. 64, 301-320 (2006)

13. Li, Y., Yao, D., Yao, J., Chen, W.: A particle swarm optimization algorithm for beam angle selection in intensity modulated radiotherapy planning. Phys. Med. Biol. 50, 3491-3514 (2005)

14. Liu, H.H., Jauregui, M., Zhang, X., Wang, X., Dongand, L., Mohan, R.: Beam angle optimization and reduction for intensity-modulated radiation therapy of non-smallcell lung cancers. Int. J. Radiat. Oncol. Biol. Phys. 65, 561-572 (2006)

15. Pugachev, A., Xing, L.: Computer-assisted selection of coplanar beam orientations in intensity-modulated radiation therapy. Phys. Med. Biol. 46, 2467-2476 (2001)

16. Pugachev, A., Xing, L.: Incorporating prior knowledge into beam orientation optimization in IMRT. Int. J. Radiat. Oncol. Biol. Phys. 54, 1565-74 (2002)

17. Rocha, A.M.A.C., Fernandes, E.M.G.P.: Modified movement force vector in an electromagnetism-like mechanism for global optimization. Opt. Methods Softw. 24, 253-270 (2009)

18. Rocha, A.M.A.C., Fernandes, E.M.G.P.: Numerical study of augmented Lagrangian algorithms for constrained global optimization. Optimization 60, 10-11 (2011)

19. Rocha, H., Dias, J.M., Ferreira, B.C., Lopes, M.C.: Beam angle optimization for intensity-modulated radiation therapy using a guided pattern search method. Phys. Med. Biol. 58, 2939-2953 (2013) 
20. Rocha, H., Dias, J.M., Ferreira, B.C., Lopes, M.C.: Selection of intensity modulated radiation therapy treatment beam directions using radial basis functions within a pattern search methods framework. J. Global Optim. 57, 1065-1089 (2013)

21. Rocha, H., Dias, J.M., Ferreira, B.C., Lopes, M.C.: Pattern search methods framework for beam angle optimization in radiotherapy design. Appl. Math. Comput. 219, 10853-10865 (2013)

22. Romeijn, H.E., Ahuja, R.K., Dempsey, J.F., Kumar, A., Li, J.: A novel linear programming approach to fluence map optimization for intensity modulated radiation therapy treatment planing. Phys. Med. Biol. 48, 3521-3542 (2003)

23. Schreibmann, E., Lahanas, M., Xing, L., Baltas, D.: Multiobjective evolutionary optimization of the number of beams, their orientations and weights for intensitymodulated radiation therapy. Phys. Med. Biol. 49, 747-770 (2004)

24. Stein, J., Mohan, R., Wang, X.H., Bortfeld, T., Wu, Q., Preiser, K., Ling, C.C., Schlegel, W.: Number and orientation of beams in intensity-modulated radiation treatments. Med. Phys. 24, 149-160 (1997) 